03

\title{
К теории взаимодействия переходного излучения заряженной частицы с периодически модулированным анизотропным магнитодиэлектрическим заполнением волновода
}

\author{
(C) Э.А. Геворкян \\ Российский экономический университет им. Г.В. Плеханова, \\ 117997 Москва, Россия \\ e-mail: gevor_mesi@mail.ru \\ Поступила в редакцию 31.10.2017 г. \\ В окончательной редакции 20.02.2018 г.
}

\begin{abstract}
Рассмотрено переходное излучение заряженной частицы в волноводе с модулированным анизотропным магнитодиэлектрическим заполнением. Предполагается, что частица движется с постоянной скоростью перпендикулярно оси волновода, заполнение которого периодически модулировано в пространстве по гармоническому закону. Получены волновые уравнения для поперечно-электрического (ТЕ) и поперечно-магнитного (ТМ) полей в волноводе. Найдены аналитические выражения для полей в волноводе в первом приближении по малым индексам модуляции заполнения волновода. Вычислены энергии переходного излучения заряженной частицы в области „слабого“ (не резонансного) взаимодействия волны излучения с волной модуляции, в частности, в случае прямоугольного волновода. Показано, что в области слабого взаимодействия энергия излучения на нулевой гармонике не зависит от индексов модуляции, а на первых гармониках она пропорциональна индексам модуляции в первой степени. Проведен анализ механизма возникновения черенковского излучения.
\end{abstract}

DOI: 10.21883/OS.2018.08.46363.258-17

\section{Введение}

Излучение Вавилова-Черенкова, возникающее при равномерном движении заряженных источников, когда их скорость движения больше фазовой скорости света в среде, впервые теоретически рассмотрено и объяснено И.Е. Таммом и И.М. Франком в 1937 г. [1]. В 1946 г. В.Л. Гинзбургом и И.М. Франком впервые был предсказан и теоретически исследован новый тип излучения движущихся заряженных источников, возникающего при их пересечении границы раздела сред с различными оптическими свойствами и названного переходным излучением [2]. В дальнейшем продолжались теоретические и экспериментальные работы для всестороннего исследования особенностей переходного излучения и излучения Вавилова-Черенкова (см., например, [3-9] и указанную в них литературу). В частности, в 1959 г. Г.М. Гарибяном в неограниченном пространстве [7] и К.А. Барсуковым в волноводе [8] теоретически было обнаружено рентгеновское переходное излучение, обладающее интересными физическими и практически используемыми свойствами.

В работе [10] решена задача переходного излучения заряженной частицы, движущейся перпендикулярно оси волновода с анизотропным магнитодиэлектрическим заполнением. В настоящей работе решается аналогичная задача в случае, когда анизотропное магнитодиэлектрическое заполнение волновода модулировано в пространстве по периодическому закону вдоль оси волновода.
Отметим, что именно наличие периодически модулированного заполнения в волноводе отличает данную работу от работы Б.М. Болотовского [6]. Подобные исследования интересны тем, что, во-первых, они способствуют развитию теории переходного излучения и излучения Вавилова-Черенкова движущихся источников в периодически модулированных средах, во-вторых, они могут открыть широкие возможности практического применения излучения источников в различных областях СВЧ электроники, микроэлектроники, тонкопленочной и интегральной оптики, физики ионосферы и радиолокационной океанографии, акустооптики и т. д. [11].

\section{Постановка задачи, волновые уравнения, поля переходного излучения}

Пусть заряженная частица с зарядом $q$ движется с постоянной скоростью $\mathbf{v}=\{v, 0,0\}$ перпендикулярно оси регулярного волновода (вдоль оси $o x$ ) с произвольным поперечным сечением, ось которого совпадает с осью $o z$ некоторой прямоугольной системы координат, пересекая поверхность волновода в точках $A_{1}\left(x_{1}, y_{0}, 0\right)$ и $A_{2}\left(x_{2}, y_{0}, 0\right)$. Предположим, что анизотропное магнитодиэлектрическое заполнение волновода модулировано по координате $z$ по периодическому закону, т.е. диэлектрическая и магнитная проницаемости заполне- 
ния имеют вид

$$
\hat{\varepsilon}=\left(\begin{array}{ccc}
\varepsilon_{1} & 0 & 0 \\
0 & \varepsilon_{1} & 0 \\
0 & 0 & \varepsilon_{2}(z)
\end{array}\right), \quad \hat{\mu}=\left(\begin{array}{ccc}
\mu_{1} & 0 & 0 \\
0 & \mu_{1} & 0 \\
0 & 0 & \mu_{2}(z)
\end{array}\right),
$$

где $\varepsilon_{1}, \mu_{1}-$ постоянные, а $\varepsilon_{2}(z)$ и $\mu_{2}(z)$ выражаются формулами

$$
\begin{gathered}
\varepsilon_{2}(z)=\varepsilon_{2}^{0}\left(1+m_{\varepsilon} \cos k_{0} z\right), \\
\mu_{2}(z)=\mu_{2}^{0}\left(1+m_{\mu} \cos k_{0} z\right) .
\end{gathered}
$$

Отметим, что в (1) $k_{0}-$ волновое число волны модуляции, индексы модуляции $m_{\varepsilon}$ и $m_{\mu}$ являются малыми параметрами $\left(m_{\varepsilon} \ll 1, m_{\mu} \ll 1, m_{\varepsilon} \approx m_{\mu}\right)$, $\varepsilon_{2}^{0}=\left.\varepsilon_{2}(z)\right|_{m_{\varepsilon}=0}, \mu_{2}^{0}=\left.\mu_{2}(z)\right|_{m_{\mu}=0}$. Как известно, плотность заряда и плотность тока в этом случае описываются с помощью $\delta$-функции Дирака и могут быть представлены в виде [12-15]

$$
\begin{gathered}
\rho=q \delta(x-v t) \delta\left(y-y_{0}\right) \delta(z), \\
j=j_{x}=q v \delta(x-v t) \delta\left(y-y_{0}\right) \delta(z) .
\end{gathered}
$$

Как и в наших ранних работах (см., например, $[10,12,16]$ ), поперечно-электрическое (ТЕ) и поперечно-магнитное (ТМ) электромагнитные поля в волноводе будем описывать с помощью продольных составляющих магнитного и электрического векторов соответственно $\left(H_{z}, E_{z}\right)$. Волновые уравнения для величин $\widetilde{H}_{z}=\mu_{2}(z) H_{z}$ и $\widetilde{E}_{z}=\varepsilon_{2}(z) E_{z}$ можно получить из уравнений Максвелла

$$
\begin{gathered}
\operatorname{rot} \mathbf{E}=-\frac{\partial \mathbf{B}}{\partial t}, \quad \operatorname{rot} \mathbf{H}=\mathbf{j}+\frac{\partial \mathbf{D}}{\partial t} \\
\operatorname{div} \mathbf{D}=\rho, \quad \operatorname{div} \mathbf{B}=0 \\
\mathbf{D}=\varepsilon_{0} \varepsilon \mathbf{E}, \quad \mathbf{B}=\mu_{0} \mu \mathbf{H},
\end{gathered}
$$

где $\varepsilon_{0}=\left(36 \pi \cdot 10^{9}\right)^{-1} \mathrm{~F} / \mathrm{m}$ - электрическая постоянная, $\mu_{0}=4 \pi \cdot 10^{-7} \mathrm{H} / \mathrm{m}$ - магнитная постоянная. Вычисления приводят к следующим волновым уравнениям в фурье-представлении:

$$
\begin{aligned}
\Delta_{\perp} \widetilde{H}_{\omega z} & +\frac{\mu_{2}(z)}{\mu_{1}} \frac{\partial^{2} \widetilde{H}_{\omega z}}{\partial z^{2}} \\
& +\varepsilon_{0} \mu_{0} \varepsilon_{1} \mu_{2}(z) \omega^{2} \widetilde{H}_{\omega z}=\mu_{2}(z) \frac{\partial j_{\omega}}{\partial y} \\
\Delta_{\perp} \widetilde{E}_{\omega z} & +\frac{\varepsilon_{2}(z)}{\varepsilon_{1}} \frac{\partial^{2} \widetilde{E}_{\omega z}}{\partial z^{2}} \\
& +\varepsilon_{0} \mu_{0} \mu_{1} \varepsilon_{2}(z) \omega^{2} \widetilde{E}_{\omega z}=\frac{\varepsilon_{2}(z)}{\varepsilon_{1} \varepsilon_{0}} \frac{\partial \rho_{\omega}}{\partial z}
\end{aligned}
$$

где $\Delta_{\perp}=\partial^{2} / \partial x^{2}+\partial^{2} / \partial y^{2}-$ двумерный оператор Лапласа, а $j_{\omega}$ и $\rho_{\omega}$ имеют вид

$$
\begin{aligned}
& j_{\omega}=\frac{1}{\sqrt{2 \pi}} q e^{i \omega \frac{x}{v}} \delta\left(y-y_{0}\right) \delta(z), \\
& \rho_{\omega}=\frac{q}{\sqrt{2 \pi} v} e^{i \omega \frac{x}{v}} \delta\left(y-y_{0}\right) \delta(z) .
\end{aligned}
$$

Решения волновых уравнений (3) и (4) будем искать в виде

$$
\begin{aligned}
\widetilde{H}_{\omega z} & =\sum_{n=0}^{\infty} \widetilde{H}_{n}(z) \hat{\Psi}_{n}(x, y), \\
\widetilde{E}_{\omega z} & =\sum_{n=0}^{\infty} \widetilde{E}_{n}(z) \hat{\Psi}_{n}(x, y),
\end{aligned}
$$

где $\hat{\Psi}_{n}(x, y)$ и $\Psi_{n}(x, y)$ - ортонормированные собственные функции второй и первой краевых задач для поперечного сечения волновода (задачи Неймана и Дирихле). Эти функции удовлетворяют следующим уравнениям Гельмгольца с соответствующими граничными условиями

$$
\begin{gathered}
\Delta_{\perp} \hat{\Psi}_{n}(x, y)+\hat{\lambda}_{n}^{2} \hat{\Psi}_{n}(x, y)=0,\left.\quad \frac{\partial \hat{\Psi}_{n}(x, y)}{\partial \mathbf{n}}\right|_{\Sigma}=0, \\
\Delta_{\perp} \Psi_{n}(x, y)+\lambda_{n}^{2} \Psi_{n}(x, y)=0, \\
\left.\Psi_{n}(x, y)\right|_{\Sigma}=0,
\end{gathered}
$$

где $\hat{\lambda}_{n}$ и $\lambda_{n}-$ собственные значения второй и первой краевых задач, $\Sigma$ - контур поперечного сечения волновода, $\mathbf{n}-$ нормаль к $\Sigma$. Аналитические выражения для поперечных составляющих ТЕ- и ТМ-полей в волноводе в фурье-представлении, полученные из уравнений Максвелла (2) с учетом (6) и (7), имеют вид:

ТЕ волна

$$
\begin{gathered}
\mathbf{H}_{\omega \tau}=\frac{1}{\mu_{1}} \sum_{n=0}^{\infty} \hat{\lambda}_{n}^{-2} \frac{d \widetilde{H}_{n}(z)}{d z} \nabla \hat{\Psi}_{n}(x, y), \\
\mathbf{E}_{\omega \tau}=-i \omega \mu_{0} \sum_{n=0}^{\infty} \hat{\lambda}_{n}^{-2} \widetilde{H}_{n}(z)\left[\mathbf{z}_{0} \nabla \hat{\Psi}_{n}(x, y)\right],
\end{gathered}
$$

ТМ волна

$$
\begin{gathered}
\mathbf{H}_{\omega \tau}=-i \varepsilon_{0} \omega \sum_{n=0}^{\infty} \lambda_{n}^{-2} \widetilde{E}_{n}(z)\left[\mathbf{z}_{0} \nabla \Psi_{n}(x, y)\right], \\
\mathbf{E}_{\omega \tau}=\frac{1}{\varepsilon_{1}} \sum_{n=0}^{\infty} \lambda_{n}^{-2} \frac{d \widetilde{E}_{n}(z)}{d z} \nabla \Psi_{n}(x, y) .
\end{gathered}
$$

Отметим, что в (8) и (9) $\Delta_{\perp}=\partial^{2} / \partial x^{2}+\partial^{2} / \partial y^{2}-$ двумерный оператор Гамильтона (набла), $\mathbf{z}_{0}-$ орт оси $o z$, индекс $\perp$ означает поперечное составляющее.

Подставляя (6) в волновые уравнения (3) и (4), производя некоторые преобразования с учетом (5) и (7), для $\widetilde{H}_{n}(z)$ и $\widetilde{E}_{n}(z)$ получим следующие линейные неоднородные обыкновенные дифференциальные уравнения второго порядка:

$$
\frac{d^{2} \widetilde{H}_{n}(z)}{d z^{2}}+\hat{\chi}_{n}^{2} \widetilde{H}_{n}(z)=-\frac{q \mu_{l}}{\sqrt{2 \pi}} \hat{B}_{n} \delta(z),
$$




$$
\frac{d^{2} \hat{E}_{n}(z)}{d z^{2}}+\chi_{n}^{2} \widetilde{E}_{n}(z)=\frac{q}{\sqrt{2 \pi} v \varepsilon_{0}} B_{n} \delta^{\prime}(z)
$$

где

$$
\begin{gathered}
\hat{\chi}_{n}^{2}=\frac{\mu_{1}}{\mu_{2}(z)}\left[\varepsilon_{0} \mu_{0} \varepsilon_{1} \mu_{2}(z) \omega^{2}-\hat{\lambda}_{n}^{2}\right], \\
\chi_{n}^{2}=\frac{\varepsilon_{1}}{\varepsilon_{2}(z)}\left[\varepsilon_{0} \mu_{0} \mu_{1} \varepsilon_{2}(z) \omega^{2}-\lambda_{n}^{2}\right], \\
\hat{B}_{n}=\left.\int_{x_{1}}^{x_{2}} \frac{\partial \hat{\Psi}_{n}(x, y)}{\partial y}\right|_{y=y_{0}} e^{\frac{i \omega x}{v}} d x, \\
B_{n}=\left.\int_{x_{1}}^{x_{2}} \Psi_{n}(x, y)\right|_{y=y_{0}} e^{\frac{i \omega x}{v}} d x .
\end{gathered}
$$

Если теперь в (12) и (13) учитывать выражения для $\varepsilon_{2}(z)$ и $\mu_{2}(z)$ (см. (1)), то в первом приближении по индексам модуляции $m_{\varepsilon}$ и $m_{\mu}$ для $\hat{\chi}_{n}^{2}$ и $\chi_{n}^{2}$ получим

$$
\begin{gathered}
\hat{\chi}_{n}^{2}=\frac{\mu_{1}}{\mu_{2}^{0}}\left[\left(\chi_{n}\right)^{2}+m_{\mu} \hat{\lambda}_{n}^{2} \cos k_{0} z\right], \\
\left(\hat{\chi}_{n}^{0}\right)^{2}=\varepsilon_{0} \mu_{0} \varepsilon_{1} \mu_{2}^{0} \omega^{2}-\hat{\lambda}_{n}^{2}, \\
\chi_{n}^{2}=\frac{\varepsilon_{1}}{\varepsilon_{2}^{0}}\left[\left(\chi_{n}^{0}\right)^{2}+m_{\mu} \lambda_{n}^{2} \cos k_{0} z\right], \\
\left(\chi_{n}^{0}\right)^{2}=\varepsilon_{0} \mu_{0} \mu_{1} \varepsilon_{2}^{0} \omega^{2}-\lambda_{n}^{2} .
\end{gathered}
$$

Переходя в волновых уравнениях (10) и (11) к новой переменной $s=\left(k_{0} z / 2\right)$ с одновременным разложением коэффициентов при $\widetilde{H}_{n}(z)$ и $\widetilde{E}_{n}(z)$ в ряд Фурье и подставляя (16)-(19) в (10) и (11), в первом приближении по $m_{\varepsilon}$ и $m_{\mu}$ (ограничиваемся рассмотрением трех пространственных гармоник) получим неоднородные дифференциальные уравнения типа Матье-Хилла [17]

$$
\begin{aligned}
& \frac{d^{2} \widetilde{H}_{n}(s)}{d s^{2}}+\left(\sum_{k=-1}^{1} \hat{\theta}_{k}^{n} e^{2 i k s}\right) \widetilde{H}_{n}(s)=-\frac{q \mu_{1}}{\sqrt{2 \pi}} \hat{B}_{n} \delta(s), \\
& \frac{d^{2} \widetilde{E}_{n}(s)}{d s^{2}}+\left(\sum_{k=-1}^{1} \hat{\theta}_{k}^{n} e^{2 i k s}\right) \widetilde{E}_{n}(s)=\frac{q}{\sqrt{2 \pi} v \varepsilon_{0}} B_{n} \delta^{\prime}(s),
\end{aligned}
$$

где

$$
\begin{gathered}
\hat{\theta}_{0}^{n}=\frac{4 \mu_{1}\left(\hat{\chi}_{n}^{0}\right)^{2}}{k_{0}^{2} \mu_{2}^{0}}, \quad \hat{\theta}_{ \pm 1}^{n}=\frac{2 \mu_{1} \hat{\lambda}_{n}^{2}}{k_{0}^{2} \mu_{2}^{0}} m_{\mu}, \\
\theta_{0}^{n}=\frac{4 \varepsilon_{1}\left(\chi_{n}^{0}\right)^{2}}{k_{0}^{2} \varepsilon_{2}^{0}}, \quad \theta_{ \pm 1}^{n}=\frac{2 \varepsilon_{1} \lambda_{n}^{2}}{k_{0}^{2} \varepsilon_{2}^{0}} m_{\varepsilon} .
\end{gathered}
$$

Решения соответствующих однородных уравнений

$$
\begin{aligned}
& \frac{d^{2} \widetilde{H}_{n}(s)}{d s^{2}}+\left(\sum_{k=-1}^{1} \hat{\theta}_{k}^{n} e^{2 i k s}\right) \widetilde{H}_{n}(s)=0, \\
& \frac{d^{2} \widetilde{E}_{n}(s)}{d s^{2}}+\left(\sum_{k=-1}^{1} \theta_{k}^{n} e^{2 i k s}\right) \widetilde{E}_{n}(s)=0,
\end{aligned}
$$

будем искать в виде

$$
\begin{aligned}
& \widetilde{H}_{n}(s)=e^{i \hat{\mu}_{n} s} \sum_{k=-1}^{1} \hat{C}_{k}^{n} e^{2 i k s}, \\
& \widetilde{E}_{n}(s)=e^{i \mu_{n} s} \sum_{k=-1}^{1} C_{k}^{n} e^{2 i k s},
\end{aligned}
$$

где характеристические числа $\hat{\mu}_{n}, \mu_{n}$ и амплитуды $\hat{C}_{k}^{n}, C_{k}^{n}$ пока неизвестные величины. Подстановка (24) и (25) в (22) и (23) приводит к дисперсионным уравнениям для определения $\hat{\mu}_{n}, \mu_{n}$ и к системам уравнений для определения $\hat{C}_{k}^{n}, C_{k}^{n}$. Решая эти уравнения в области слабого (не резонансного) взаимодействия между волной излучения и волной модуляции $\left(\hat{\theta}_{0}^{1} \neq 1, \theta_{0}^{n} \neq 1\right)$, для величин $\hat{\mu}_{n}, \mu_{n}$ и $\hat{C}_{k}^{n}, C_{k}^{n}$ в первом приближении по индексам модуляции получим

$$
\begin{gathered}
\left(\hat{\mu}_{n}\right)^{2} \simeq \hat{\theta}_{0}^{n}, \quad\left(\mu_{n}\right)^{2} \simeq \theta_{0}^{n}, \\
\hat{C}_{ \pm 1}^{n} \cong \frac{\hat{\theta}_{1}^{n} \hat{C}_{0}^{n}}{4\left(1 \pm \sqrt{\hat{\theta}_{0}^{n}}\right)}, \quad C_{ \pm}^{n} \cong \frac{\theta_{1}^{n} C_{0}^{n}}{4\left(1 \pm \sqrt{\theta_{0}^{n}}\right)},
\end{gathered}
$$

где величины $\hat{C}_{0}^{n}$ и $C_{0}^{n}$ можно найти из условия нормировки.

Пользуясь методом вариации произвольных постоянных для определения частных решений неоднородных уравнений (20), (21) и учитывая условия излучения (нет волн, распространяющихся к источнику излучения) для $\widetilde{H}_{n}(z)$ и $\widetilde{E}_{n}(z)$, получим

$$
\begin{array}{r}
\widetilde{H}_{n}(z)=\frac{i \xi_{n}}{\hat{C}_{0}^{n} \hat{\mu}_{n} k_{0}} \sum_{k=-1}^{1} \hat{C}_{k}^{n} \cos \frac{k_{0}\left(\hat{\mu}_{n}+2 k\right) z}{2}, \\
\widetilde{E}_{n}(z)=\frac{k_{0} \xi_{n}}{2 C_{0}^{n}} \sum_{k=-1}^{1} C_{k}^{n} \sin \frac{k_{0}\left(\mu_{n}+2 k\right) z}{2},
\end{array}
$$

где

$$
\xi_{n}=-\frac{q \mu_{1}}{\sqrt{2 \pi}} \hat{B}_{n}, \quad \xi_{n}=\frac{q}{\sqrt{2 \pi} \cdot \varepsilon_{0} v} B_{n} .
$$

(26) и (27) указывают на то, что поперечно-электрическое (ТЕ) и поперечно-магнитное (ТМ) поля переходного излучения заряженной частицы, движущейся перпендикулярно оси волновода с периодически модулированным анизотропным магнитодиэлектрическим заполнением представляют сумму пространственных гармоник с различными амплитудами. При этом на основной гармонике $(k=0)$ амплитуды не зависят от индексов модуляции, а на боковых гармониках $(k= \pm 1)$ они пропорциональны индексам модуляции в первой степени. 


\section{Потери энергии на переходное излучение}

Энергии переходного излучения движущейся частицы на ее траектории от $x_{1}$ до $x_{2}$ можно найти с помощью тормозящей силы $q E_{\omega x}$, действующей на частицу со стороны создаваемого ею поля. Вычисления приводят к следующим выражениям:

$$
\begin{aligned}
& S_{n}^{T E}=\frac{\mu_{0} \sqrt{\mu_{2}^{0} \mu_{1}} q^{2}}{4 \pi \hat{\lambda}_{n}^{2}} \\
& \times \operatorname{Re} \int_{0}^{\infty}\left[1+\frac{\mu_{1} \hat{\lambda}_{n}^{2}}{k_{0}^{2} \mu_{2}^{0}-4 \mu_{1}\left(\hat{\chi}_{n}^{0}\right)^{2}} m_{\mu}\right] \frac{\omega}{\hat{\chi}_{n}^{0}}\left|\hat{B}_{n}\right|^{2} d \omega, \\
& S_{n}^{T M}=\frac{k_{0} q^{2}}{2 \pi \varepsilon_{0} \sqrt{\varepsilon_{1} \varepsilon_{2}^{0}} \lambda_{0}^{2} v^{2}} \\
& \quad \times \operatorname{Re} \int_{0}^{\infty} i\left[1+\frac{3 \varepsilon_{1} \lambda_{n}^{2}}{k_{0}^{2} \varepsilon_{2}^{0}-4 \varepsilon_{1}\left(\chi_{n}^{0}\right)^{2}} m_{\varepsilon}\right] \omega\left|B_{n}\right|^{2} d \omega,
\end{aligned}
$$

где из области интегрирования исключаются частоты, удовлетворяющие условиям

$$
\begin{aligned}
& k_{0}^{2} \mu_{2}^{0}-4 \mu_{1}\left(\hat{\chi}_{n}^{0}\right)^{2}=0, \\
& k_{0}^{2} \varepsilon_{2}^{0}-4 \varepsilon_{1}\left(\chi_{n}^{0}\right)^{2}=0 .
\end{aligned}
$$

Как следует из (28) и (29), модуляция заполнения волновода приводит к тому, что в формулах для энергии излучения под интегралами появляются члены, пропорциональные индексам модуляции в первой степени.

Теперь перейдем к рассмотрению частного случая прямоугольного волновода, стенки которого определяются уравнениями $x=0, x=a, y=0, y=b$. Как известно, ортонормированные собственные функции $\hat{\Psi}_{n}(x, y), \Psi_{n}(x, y)$ и собственные значения $\hat{\lambda}_{n}, \lambda_{n}$ краевых задач Неймана и Дирихле для поперечного сечения волновода выражаются формулами [18]

$$
\begin{gathered}
\hat{\Psi}_{n}(x, y)=\hat{\Psi}_{n, m}(x, y)=\sqrt{\frac{\delta_{n} \delta_{m}}{a b}} \cos \frac{\pi m}{a} x \cos \frac{\pi n}{b} y, \\
\Psi_{n}(x, y)=\Psi_{n, m}(x, y)=\sqrt{\frac{4}{a b}} \sin \frac{\pi m}{a} x \sin \frac{\pi n}{b} y, \\
\hat{\lambda}_{n}=\hat{\lambda}_{n, m}=\lambda_{n}=\lambda_{n, m}=\pi \sqrt{\frac{m^{2}}{a^{2}}+\frac{n^{2}}{b^{2}}}, \\
\delta_{i}=2, \quad i \neq 0, \quad \delta_{0}=1 .
\end{gathered}
$$

Проводя интегрирование по $x$ от 0 до $a$ в выражениях для $\hat{B}_{n}$ и $B_{n}$ (см. (14) и (15)) с учетом (30)-(32) и подставляя в $(28)$ и (29), для $S_{n}^{(T E)}$ и $S_{n}^{(T M)}$ получим

$$
\begin{aligned}
S_{n, m}^{(T E)}= & \frac{\mu_{0} \sqrt{\mu_{2}^{0} \mu_{1}} q^{2} \pi n^{2} \delta_{n} \delta_{m} k_{0}^{2} \sin ^{2}\left(\frac{\pi n}{b} y_{0}\right)}{a b^{3} v^{2} \hat{\lambda}_{n, m}^{2}} \\
\times & \operatorname{Re} \int_{0}^{\infty}\left(1+\frac{\mu_{1} \hat{\lambda}_{n, m}^{2}}{k_{0}^{2} \mu_{2}^{0}-4 \mu_{1}\left(\hat{\chi}_{n, m}^{0}\right)^{2}} m_{\mu}\right) \frac{\omega^{3}}{\hat{\chi}_{n, m}^{0}} \\
\times & \frac{\sin ^{2}\left[\left(\frac{\pi m}{a}-\frac{\omega}{v}\right) \frac{a}{2}\right]}{\left[\left(\frac{\pi m}{a}\right)^{2}-\frac{\omega^{2}}{v^{2}}\right]^{2}} d \omega, \\
S_{n, m}^{(T M)}= & \frac{8 \pi k_{0} q^{2} m^{2} \sin ^{2}\left(\frac{\pi n}{b} y_{0}\right)}{\varepsilon_{0} \sqrt{\varepsilon_{1} \varepsilon_{2}^{0}} a^{3} b v^{2} \hat{\lambda}_{n, m}^{2}} \\
& \times \operatorname{Re} \int_{0}^{\infty} i\left(1+\frac{3 \varepsilon_{1} \lambda_{n, m}^{2}}{k_{0}^{2} \varepsilon_{2}^{0}-4 \varepsilon_{1}\left(\chi_{n, m}^{0}\right)^{2}} m_{\varepsilon}\right) \\
& \times \frac{\sin ^{2}\left[\left(\frac{\pi m}{a}-\frac{\omega}{v}\right) \frac{a}{2}\right]}{\left[\left(\frac{\pi m}{a}\right)^{2}-\frac{\omega^{2}}{v^{2}}\right]^{2}} \omega d \omega,
\end{aligned}
$$

Как следует из (33) и (34) (см. также [13]), в излучении могут отсутствовать моды с индексом $n$, которые удовлетворяют условию $\sin ^{2}\left(\pi n y_{0} / b\right)=0, n y_{0} / b=r$, $r=0,1,2,3, \ldots$. Если, в частности, $r=1$, то имеем $n y_{0}=b$. Это условие указывает на то, что отсутствует взаимодействие между заряженной частицей и соответствующей модой (частица проходит через узел продольной составляющей электрического поля). Кроме того, в излучении будут отсутствовать частоты, удовлетворяющие условию

$$
\frac{\omega a}{2 v}-\frac{\pi m}{2}=p \pi, \quad p=1,2,3, \ldots,
$$

которое можно переписать в виде

$$
\frac{a}{\pi v / \omega}=m+2 p
$$

Теперь, если учесть, что $\pi v / \omega$ есть половина длины волны тока, создаваемого частицей, то из (35) можно сделать вывод, что когда на траектории частицы, равной $a$, укладывается целое число полуволн тока, то из-за интерференции излучение с такой частотой отсутствует. Это утверждение не распространяется на случай, когда $p=0$, так как при этом $(\omega a / 2 v)-(\pi m / 2)=0$ и под интегралами в (33) и (34) подынтегральные функции имеют устранимый разрыв на частоте $\omega=\pi m v / a$. Здесь опять на траектории частицы укладывается целое число полуволн тока частицы. Но если раньше излучение с такой частотой гасилось из-за интерференции от отдельных частей траектории частицы, то при $p=0$ на частоте $\omega=\pi m v / a$ начинает работать новый механизм 
излучения. Оказывается, что $\omega=\pi m v / a$ и есть частота черенковского излучения и подынтегральная функция

$$
\frac{\sin ^{2}\left[\left(\frac{\pi m}{a}-\frac{\omega}{v}\right) \frac{a}{2}\right]}{\left[\left(\frac{\pi m}{a}\right)^{2}-\left(\frac{\omega}{v}\right)^{2}\right]^{2}}
$$

при

$$
\omega_{\mathrm{Cher}}=\frac{\pi m v}{a}
$$

имеет устранимый разрыв и принимает значение, пропорциональное $a^{2}$ (квадрат длины траектории частицы). При вычислении энергии излучения ВавиловаЧеренкова интегралы в (33) и (34) берутся по окрестности $\omega_{\text {Cher }}$ размер которой определяется из соотношения

$$
\begin{gathered}
\left.\frac{a}{2} \frac{d}{d \omega}\left(\frac{\omega}{v}-\frac{\pi m}{a}\right)\right|_{\omega=\omega_{\text {Cher }}} \Delta \omega_{\text {Cher }} \cong 2 \pi, \\
\Delta \omega_{\text {Cher }} \cong \frac{4 \pi v}{a}
\end{gathered}
$$

Механизм возникновения излучения Вавилова-Черенкова при выполнении условия его возникновения $\left(\varepsilon_{0} \mu_{0} \varepsilon_{1} \mu_{2}^{0} v^{2}>1\right.$ для ТЕ-волны, $\varepsilon_{0} \mu_{0} \mu_{1} \varepsilon_{2}^{0} v^{2}>1$ для ТМ-волны) заключается в том, что заряженная частица во время своего движения взаимодействует с сопутствующим ей полем в стоячей волне в плоскости хоу и фазовая скорость сопутствующей волны становится равной скорости частицы, т.е.

$$
v_{n, m, \mathrm{ph}}=\frac{\omega_{\mathrm{Cher}}}{k}=v
$$

где $k=\pi m / a$ есть волновое число этой волны. Для получения представления о порядке величины частоты излучения Вавилова-Черенкова по формуле (36) рассмотрим следующий пример. Пусть $\varepsilon_{1} \simeq \varepsilon_{2}^{0} \simeq 2.6$, $\mu_{1} \simeq \mu_{2}^{0} \simeq 2.1, \quad m=1, \quad v \simeq 2 \cdot 10^{8} \mathrm{~m} / \mathrm{s} \quad$ (такую скорость имеет частица при энергии $7 \cdot 10^{5} \mathrm{eV} \simeq 10^{-13} \mathrm{~J}$ ) [5]. Тогда при $a=0.8 \cdot 10^{-3} \mathrm{~m}$ по формуле (35) получим $\omega_{\text {Cher }} \simeq 7.85 \cdot 10^{11} \mathrm{~Hz}$, а при $a=10^{-3}$ получим $\omega_{\text {Cher }} \simeq$ $\simeq 6.28 \cdot 10^{22} \mathrm{~Hz}$. Отметим, что полученные оценки частоты излучения Вавилова-Черенкова по порядку совпадают со значением, полученным в работе [9].

\section{Заключение}

Полученные в работе результаты показывают, что пространственная периодическая модуляция заполнения волновода существенным образом влияет на процесс переходного излучения. Поля в волноводе представляют собой набор пространственных гармоник с различными амплитудами, при этом если на нулевой гармонике поля не зависят от индексов модуляции, то на плюс и минус первых гармониках они пропорциональны индексам модуляции в первой степени. В выражениях для энергий переходного излучения под интегралами добавляются члены, пропорциональные индексам модуляции в первой степени. В области слабого взаимодействия излучения с модулированным заполнением на определенной частоте появляется излучение Вавилова-Черенкова, что выражается резким пиком в спектре переходного излучения. Ширина частотной области черенковского излучения прямо пропорциональна скорости движения частицы и обратно пропорциональна длине траектории частицы.

\section{Список литературы}

[1] Гинзбург В.Л., Франк И.М. // ЖЭТФ. 1946. В. 16. С. 15-28.

[2] Тамм И.Е., Франк И.М. // ДАН СССР. 1937. Т. 14. В. 3. C. $107-112$.

[3] Гинзбург В.Л. // УФН. 1996. Т. 166. № 10. С. 1033-1042.

[4] Болотовский Б.М., Серов Ф.В. // УФН. 2009. Т. 179. № 5. C. $517-524$.

[5] Болотовский Б.М. // УФН. 2009 Т. 179. № 11. C. $1161-1173$.

[6] Болотовский Б.М. // УФН. 1961. Т. 75. В. 2. С. 295-350.

[7] Гарибян Г.М. // ЖЭТФ. 1959. Т. 37. В. 2(8). С. 527-533.

[8] Барсуков К.А. // ЖЭТФ. 1959. Т. 37. В. 4(10). C. $1106-1109$.

[9] Cook A.M., Tikhoplav R., Tochitsky S.Y., Travish G., Williams O.B., Rosenzweig J.B. // Phys. Rev. Lett. 2009. V. 103. P. 095003-1-095003-4.

[10] Геворкян Э.А. // Опт. и спектр. 2015. Т. 119. № 2. C. 302-306; Gevorkyan E.A. // Opt. Spectrosc. 2015. V. 119. N 2. P. $286-290$.

[11] Болотовский Б.М. // УФН. 2009. Т. 179. № 11. С. 517-524.

[12] Геворкян Э.А. // Успехи современной радиоэлектроники. 2006. № 1. C. 3-29.

[13] Барсуков К.А., Газазян Э.Д., Лазиев Э.М. // Известия вузов. Радиофизика. 1972. Т. 15. № 2. С. 191-195.

[14] Gevorkyan E.A. // Proc. 16th International Conference on Mathematical Methods in Electromagnetic Theory. Lviv. Ukraine. 2016. P. 334-336.

[15] Gevorkyan E.A., Tatarnikov O.V. // Proc. 21st International Seminar/Workshop on Direct and Inverse Problems of Electromagnetic and Acoustic Wave Theory. Tbilisi. Georgia. 2016. P. 17-19.

[16] Gevorkyan E.A. // Wave propagation / Ed. by Petrin A., IntechOpen, 2011. Р. 267; [Электронный ресурс] Режим доступа: http://www.intechopen.com

[17] Уитеккер Э.Г., Ватсон Дж.Р. Курс современного анализа. Пер. с англ. В 2 частях. М.: URSS, 2015. 864 с.

[18] Тихонов А.Н., Самарский А.А. Уравнения математической физики. М.: МГУ им. М.В. Ломоносова, 2004. 798 с. 